\title{
Positive Regulation of Angiogenesis
}

National Cancer Institute

\section{Source}

National Cancer Institute. Positive Regulation of Angiogenesis. NCI Thesaurus. Code C96323.

A process or mechanism that promotes, initiates or sustains angiogenesis. 